\title{
Adherence towards COVID-19 prevention measures and associated factors in Hossana town, South Ethiopia, 2021
}

\author{
Temesgen Fonkamo ${ }^{1}$ and LONSAKO WOILORO ${ }^{1}$ \\ ${ }^{1}$ Wachemo University
}

April 27, 2021

\begin{abstract}
Introduction: The covid-19 disease is a pandemic threat for humanity's healthcare system, social, economic, and psychological well-being for both developed and developing nations. In the case of developing nations such as the resource of Ethiopia, however, the key obstacle is to buy the vaccine and administer it to their people.. In the study area, however, the degree of adherence to the covid-19 preventive measure was not well established. The aim of this study is to determine adherence to covid-19 prevention measures in Hossana town. Methods: From 3 to 29 January 2021, a community-based cross-sectional study was conducted among individuals living in the Hosanna town. We used a sample size of 384 . The sample size was distributed to all 8 kebeles in proportion to the size of the households contained in each kebele in the town of Hossana. Systematic sampling methods were used and both descriptive and advanced analysis, data was entered into Epi-data and exported to SPSS. Binary logistic regression was used to identify variables associated with adherence to preventive measures for covid-19. Result: $50.4 \%$ of the study participants had good adherence with the COVID-19 preventive measures. 145 (38.5\%) of all respondents had poor knowledge on COVID-19 preventive measures and $40.3 \%$ had poor COVID-19 transmission methods knowledge. Age [AOR: 0.34; $95 \%$ CI (0.131-0.912)], educational status [AOR: 0.32; 95\% CI (0.165-0.632)], marital status [AOR: 2; \% CI (1.191-3.803)], family size [AOR: 2.4; \% CI (1.322-4.366)]] and covid-19 complication [AOR: 0.49: 95\% CI (0.242-0.979)]] were significantly associated with covid-19 prevention measurement adherence in multivariate analysis. Conclusion: This study found that approximately half of the participants had poor adherence to COVID-19 preventive measures. Factors associated with covid-19 preventive measures were age, educational age, marital status, family size, and heard about complication of COVID-19 were associated with preventive measures.
\end{abstract}

\section{Hosted file}

covid -19 manuscrpt.pdf available at https://authorea.com/users/410329/articles/519745adherence-towards-covid-19-prevention-measures-and-associated-factors-in-hossana-townsouth-ethiopia-2021 